\title{
Some Consequences of Infinite Mass Renormalization
}

\author{
WOLFHART ZIMMERMANN \\ Courant Institute of Mathematical Sciences
}

Received December 20, 1967

\begin{abstract}
Using a finite form of local field equations some consequences of infinite mass renormalization are studied in a rigorous manner. The method is applied to various models. For pseudoscalar meson-nucleon interaction sufficient conditions are given for the equivalence to a direct Fermi coupling. Confirming a recent result by KROLL, LEE, and ZUMINo it is shown that a vector meson field should be proportional to the corresponding current if the bare meson mass is infinite. In the conventional treatment of neutral vector meson theories this causes certain difficulties which are analyzed in detail. In case of two vector fields coupled to the same current it is found that the fields must be proportional provided the bare masses are both infinite. In the Appendix finite local field equations are discussed for the coupling of a neutral vector meson field to the current of a spin 1/2 field.
\end{abstract}

\section{Introduction}

To illustrate the purpose of this paper we consider the model of $A^{4}$ coupling. The model is described by a scalar, Hermitian field $A(x)$ which is an operator valued distribution

$$
A_{f}=\int f(x) A(x) d x
$$

defined for a suitable class of test functions on a dense linear subset $D$ of Hilbert space. $A(x)$ is supposed to satisfy the general principles in Wightman's formulation of relativistic, local quantum field theory $[1-3]^{1}$. In addition we assume that $A(x)$ satisfies the field equation ${ }^{2} 3$

$$
\begin{gathered}
-\left(\square+m^{2}\right) A(x)=\lim _{\xi \rightarrow 0} j(x \xi) \\
j(x, \xi)=\lambda Z_{1}(\xi) Z_{2}^{-1}(\xi): A(x+\xi) A(x) A(x-\xi):-\delta m^{2}(\xi) A(x)
\end{gathered}
$$

which has been verified in renormalized perturbation theory [8]. The : :product is defined by the usual vacuum subtractions

$$
\begin{aligned}
& : A\left(x_{1}\right) A\left(x_{2}\right) A\left(x_{3}\right): \\
& \quad=A\left(x_{1}\right) A\left(x_{2}\right) A\left(x_{3}\right)-\left\langle A\left(x_{1}\right) A\left(x_{2}\right)\right\rangle_{0} A\left(x_{3}\right)-\text { cycl. perm. }
\end{aligned}
$$

for

$$
\left(x_{i}-x_{j}\right)^{2}<0 \quad(i \neq j) .
$$

1 Concerning the choice of the class of test functions see JAFFE's discussion [3].

2 Field equations of this type were first proposed by VaLatin [4] for quantum electrodynamics. The theory has been further developed and extended to other interactions in ref. [5-9].

3 Throughout this paper $\lim _{\xi \rightarrow 0}$ will denote the spacelike limit with $\xi^{2}<0$ and $\xi^{\mu} / \sqrt{-\xi^{2}}$ bounded. 
$m$ and $\lambda$ denote the (finite) mass and coupling constants. Formally the values of the functions $Z_{1}, Z_{2}$ and $\delta m^{2}$ at $\xi=0$ represent the renormalization constants of vertex function, wave function and mass respectively ${ }^{4}$.

The limit $\xi \rightarrow 0$ should be understood in the weak sense that (1.1) holds for matrix elements

$$
\left(\Phi_{1}, \ldots \Phi_{2}\right) \text { with } \Phi_{1} \in D_{1}, \Phi_{2} \in D_{2} .
$$

$D_{1}$ and $D_{2}$ are dense linear subsets of $D$ with the property that

$$
\left(\Phi_{1}, A(x) \Phi_{2}\right),\left(\Phi_{1}, A(x+\xi) A(x) A(x-\xi) \Phi_{2}\right)
$$

are ordinary functions of $x$ and $\xi$ for

$$
\Phi_{1} \in D_{1}, \Phi_{2} \in D_{2} \text { and } \xi^{2}<0 \text {. }
$$

We will derive a simple consequence of the assumption that the mass renormalization is infinite, i.e.

$$
\left|\lim _{\xi \rightarrow 0} \delta m^{2}(\xi)\right|=\infty
$$

Equ. (1.1) may be written in the equivalent form

$$
-\left(\square+m^{2}\right) A(x)=j(x, \xi)+o(x, \xi)
$$

where

$$
\lim _{\xi \rightarrow 0} o(x, \xi)=0 \text {. }
$$

Dividing both sides of (1.5) by $\delta m^{2}(\xi)$ and taking the limit $\xi \rightarrow 0$ we obtain with (1.4) the relation

$$
A(x)=\lambda \lim _{\xi \rightarrow 0} \frac{Z_{1}(\xi): A(x+\xi) A(x) A(x-\xi):}{Z_{2}(\xi) \delta m^{2}(\xi)}
$$

for matrix elements of the form (1.3). As should be clear from the derivation equ. (1.6) is an identity which follows from the field equation provided the mass renormalization is infinite ${ }^{5}$. Moreover, (1.6) must be valid in perturbation theory.

4 In terms of the functions $\alpha, \beta, \gamma$ of ref. [8] the functions $Z_{1}, Z_{2}$ and $\delta m^{2}$ are defined by

$$
\begin{aligned}
Z_{1}(\xi) & =\gamma(\xi)^{-1}, Z_{2}(\xi)=1+\frac{\beta(\xi)}{\gamma(\xi)} \\
\delta m^{2}(\xi) & =\lambda \frac{\alpha(\xi)}{\beta(\xi)+\gamma(\xi)} .
\end{aligned}
$$

5 It should be noted that (1.6) does not imply the relation

$\left(^{*}\right)[A(x) A(y)]=\lambda \lim _{\xi \rightarrow 0}\left[\frac{Z_{1}(\xi): A(x+\xi) A(x) A(x-\xi):}{Z_{2}(\xi) \delta m^{2}(\xi)}, \dot{A}(y)\right], x^{0}=y^{0}$

which would be inconsistent with the canonical commutation relations.

${ }^{*}$ ) does not follow from (1.6) because (1.6) is restricted to matrix elements (1.3) for the field equation (1.1) is correct. For instance, in order to derive the vacuum expectation value of $\left(^{*}\right)$ one would need (1.1) for matrix elements

$$
\begin{aligned}
& (\Omega, \ldots \phi) \text { and }(\phi, \ldots \Omega) \\
& \phi=\int d \vec{y} \dot{A}(y) f(\vec{y}) \Omega .
\end{aligned}
$$

But even if $Z_{2}^{-1}$ were finite the left hand side of (1.1) would not be defined for (**) unless $\int x^{2} \varrho\left(\varkappa^{2}\right) d \varkappa^{2}<\infty$ ( $\varrho$ denotes the weight function of the propagator). 
The present paper will be concerned with polynomial field relations of type (1.6) for various models.

We briefly review some previous papers dealing with polynomial field relations. Usually the relations are formally stated as

$$
A(x)=P\left(A(x), A_{1}(x), \ldots, A_{n}(x)\right)
$$

where $P$ is a polynomial in the components of fields $A, A_{1}, \ldots A_{n}$ (and their spatial derivatives) occurring in a Lagrange formulation of a local and relativistic theory. If $P$ does not depend on $A$ (1.7) may be used for eliminating the field $A$. It has recently been claimed [10] that the possibility of expressing a field $A$ by a polynomial (1.7) is

(i) equivalent to the condition $Z_{A}=0, Z_{A}$ being the wave function renormalization constant of the field $A$,

(ii) equivalent to the compositeness of the particles associated with $A$.

Concerning (i) BraNDT, Sucher and Woo [11] point out that $Z_{A}=0$ should not be expected to be a necessary condition. For the Lee model they have shown that the $V$-field may be expressed in terms of the $N$ and $\theta$-field in a situation where $Z_{V} \neq 0$, but $\delta m_{V}^{2}=\infty$, in accordance with the argument leading to (1.6).

With regard to (ii) we remark that polynomials (1.7) may certainly be used to construct local field operators for composite particles. But a polynomial relation of the form (1.7) does not necessarily indicate that a composite particle is associated with the field $A$. Equation (1.6), for instance, can hardly be related to a composite structure considering that it is valid in perturbation theory.

In two recent papers $[12,13]$ it was recognized that in some cases the divergence of the self-mass is the relevant condition for (1.7). Making formal use of the field equation HAGEN [12] derived polynomial field relations from the condition of infinite self-mass. In a similar way KroLL, LEE and ZUMTNo [13] arrived at the conclusion that a vector meson field should be proportional to the corresponding current provided the bare mass of the meson is infinite.

There are, however, differences in interpretation. HAGEN argues that for infinite self-mass the equation of motion degenerates into a constraint, i.e. the field equation becomes meaningless and is replaced by a polynomial relation. In contradistinction KROLL, LEE and ZuMINo maintain that field equation and the proportionality of field and current are valid simultaneously.

The rigorous argument given above for the $A^{4}$-coupling makes evident that the interpretation given by KRoLL, LEE and ZUMrNo is the correct one. For the polynomial relation (1.6) is a simple consequence of the field equation (1.1) if the self-mass diverges in the sense of (1.4). In that case field equation and polynomial field relation hold simultaneously. 
The principal difficulty in such problems is that the field equations in their usual form become meaningless if some of the parameters are infinite. Ambiguities are likely to appear since there is no algorithm for infinite quantities. However, the limit form of field equations - such as (1.1) - is well defined even if the renormalization constants should be infinite. On this basis polynomial field relations may be derived and studied in a rigorous manner.

We finally mention a paper by NISHIJIMA [14] in which a method is developed for checking the limit form of (1.7) directly in perturbation theory. As sufficient for (1.7) NISHIJIMA finds the stronger condition that the self-mass associated with the field $A$ should diverge more strongly than any corresponding vertex renormalization. This condition would not suffice in case of a neutral vector field coupled to a conserved current since the self-mass of the vector meson is only logarithmically divergent. However, a corrected version of NISHIJIMA's argument leads for the $A^{4}$ coupling to the same result that the divergence of the self-mass should be sufficient for the polynomial field relation (1.6).

The contents of the present paper is as follows. In section 2 polynomial field relations are discussed for the meson-nucleon interaction. Divergence of the meson self-mass implies an expression for the meson field which involves a $\bar{\psi} \gamma_{5} \psi$ and an $A^{3}$-term - due to the presence of the $A^{4}$-coupling. Under more restrictive conditions, however, it can be shown that the meson field may be expressed in terms of the nucleon field only

$A(x)=\lambda g \lim _{\xi \rightarrow 0} F(\xi) \frac{1}{2}\left(: \bar{\psi}(x+\xi) \gamma^{5} \psi(x-\xi):+: \bar{\psi}(x-\xi) \gamma^{5} \psi(x+\xi):\right)$

Inserting (1.8) into the nucleon field equation one finds an equation for $\psi$ alone which formally corresponds to a Lagrangian with a direct Fermi coupling. Under different conditions such an equivalence has first been stated by Jouver [15] on the basis of formal arguments.

Section 3 and 5 concern interactions of vector meson fields. Confirming the argument of KROLL, LEE and Zumivo it will be shown that a vector meson field $V^{\mu}$ is proportional to the corresponding current $j^{\mu}$

$$
V^{\mu}=\varrho j^{\mu}
$$

provided the meson self-mass is infinite.

In section 4 this result is applied to the commutators of fields and currents in a neutral vector meson theory. It is found that the usual assumption of

$$
\left[j^{0}(x), V^{k}(y)\right]=0 \text { for } x^{0}=y^{0}
$$

is not correct if the meson mass renormalization is infinite. (1.10) is the basic hypothesis in JoHnson's derivation [16] of the sum rule

$$
\frac{1}{Z_{3} m_{0}^{2}}=\frac{1}{m^{2}}+\int \frac{\varrho\left(x^{2}\right)}{\varkappa^{2}} d \varkappa^{2} \text {. }
$$


However, the sum rule (1.11) is valid in general and can be proved by a method due to SYmanzIK [17]. SymanzIK's method also provides the complete set of equal time commutators of the meson field components and their time derivatives. Combined with (1.9) one further obtains the equal time commutators of the current components.

In section 6 the case of two vector meson fields coupled to the same current is considered. It is shown that both fields must be proportional if their masses are infinite. Furthermore the bare masses $m_{01}, m_{02}$ are equal in the sense

$$
\lim _{\xi \rightarrow 0} \frac{m_{01}^{2}(\xi)}{m_{02}^{2}(\xi)}=1 \text {. }
$$

This result shows that a system of two independent vector meson fields coupled to the same current cannot be quantized in a consistent manner if the bare masses of the fields are infinite.

The Appendix contains a detailed discussion of the local field equations in limit form for a neutral vector meson field coupled to the current of a charged spin $1 / 2$ field.

\section{Equivalence of Pseudoscalar Meson-Nucleon Interaction to a Direct Fermi Coupling}

For the model of pseudo-scalar meson-nucleon interaction the following field equations have been verified in renormalized perturbation theory $[6,9]$

$$
\begin{gathered}
\left(-i \gamma^{\mu} \partial_{\mu}+M\right) \psi(x)=h(x) \\
h(x)=\lim _{\xi \rightarrow 0}\left\{\frac{g}{2} Z_{g 1}(\xi) Z_{2}(\xi)^{-1}(A(x+\xi)\right. \\
\left.+A(x-\xi)) \gamma_{5} \psi(x)+\delta M(\xi) \psi(x)\right\} \\
-\left(\square+m^{2}\right) A(x)=j(x) \\
j(x)=\lim _{\xi \rightarrow 0}\left\{g Z_{g 1}(\xi) Z_{3}(\xi)^{-1} \varphi(x \xi)\right. \\
\left.+\lambda Z_{\lambda 1}(\xi) Z_{3}(\xi)^{-1} B(x \xi)-\delta m^{2}(\xi) A(x)\right\}
\end{gathered}
$$

with

$$
\begin{gathered}
\varphi(x \xi)=\frac{1}{2}: \bar{\psi}(x+\xi) \gamma^{5} \psi(x-\xi):+\frac{1}{2}: \bar{\psi}(x-\xi) \gamma^{5} \psi(x+\xi): \\
B(x \xi)=: A(x+\xi) A(x) A(x-\xi): \\
: \bar{\psi}\left(x_{1}\right) \gamma^{5} \psi\left(x_{2}\right):=\bar{\psi}\left(x_{1}\right) \gamma^{5} \psi\left(x_{2}\right)-\left\langle\bar{\psi}\left(x_{1}\right) \gamma^{5} \psi\left(x_{2}\right)\right\rangle_{0} \\
\text { for } \quad\left(x_{1}-x_{2}\right)^{2}<0 .
\end{gathered}
$$

Equ. (2.1-2) hold for matrix elements between suitable state vectors.

As in the case of $A^{4}$-coupling (Section 1) one obtains from the field equations $(2.1-2)$ the relations

$$
\psi(x)=-\frac{g}{2} \lim _{\xi \rightarrow 0} \frac{Z_{g 1}(\xi)(A(x+\xi)+A(x-\xi)) \gamma_{5} \psi(x)}{Z_{2}(\xi) \delta M(\xi)}
$$


if

and

$$
\left|\lim _{\xi \rightarrow 0} \delta M(\xi)\right|=\infty
$$

if

$$
A(x)=\lim _{\xi \rightarrow 0} g \frac{Z_{01}(\xi) \varphi(x \xi)+\lambda Z_{\lambda_{1}}(\xi) B(x \xi)}{Z_{3}(\xi) \delta m^{2}(\xi)}
$$

Equ. (2.8) cannot be used to eliminate the meson field since the right hand side still involves $A$. In order to express $A$ in terms of the nucleon field only we will use some additional information. In perturbation theory [9] the meson current (2.2) can be decomposed into two independent currents which are separately finite ${ }^{6}$

$$
\begin{aligned}
j(x) & =j_{1}(x)+j_{2}(x) \\
j_{1}(x) & =\lim _{\xi \rightarrow 0}\left\{g a_{11}(\xi) \varphi(x \xi)+g a_{12}(\xi) B(x \xi)-\delta m_{1}^{2}(\xi) A(x)\right\} \\
j_{2}(x) & =\lim _{\xi \rightarrow 0}\left\{\lambda a_{21}(\xi) \varphi(x \xi)+\lambda a_{22}(\xi) B(x \xi)-\delta m_{2}^{2}(\xi) A(x)\right\} .
\end{aligned}
$$

The two currents are independent in the sense

$$
\begin{gathered}
\lim _{\xi \rightarrow 0} \operatorname{det}\left(a_{i{ }_{k}}(\xi)\right) \neq 0 \\
\lim _{\xi \rightarrow 0}\left|\begin{array}{l}
g a_{12}(\xi) \delta m_{1}^{2}(\xi) \\
\lambda a_{22}(\xi) \delta m_{2}^{2}(\xi)
\end{array}\right| \neq 0 \\
\lim _{\xi \rightarrow 0}\left|\begin{array}{l}
g a_{11}(\xi) \delta m_{1}^{2}(\xi) \\
\lambda a_{21}(\xi) \delta m_{2}^{2}(\xi)
\end{array}\right| \neq 0 .
\end{gathered}
$$

Under the additional hypothesis (2.10-13) the field $A$ may be expressed in terms of $\psi, \bar{\psi}$ provided certain linear combinations of the mass renormalization constants $\delta m_{1}^{2}, \delta m_{2}^{2}$ are infinite. For the proof we write $(2.11-12)$ in the equivalent form

with

$$
\begin{aligned}
& j_{1}=g a_{11} \varphi+g a_{12} B-\delta m_{1}^{2} A+o_{1} \\
& j_{2}=\lambda a_{21} \varphi+\lambda a_{22} B-\delta m_{2}^{2} A+o_{2}
\end{aligned}
$$

$$
\lim _{\xi \rightarrow 0} o_{k}(x \xi)=0 \text {. }
$$

From these equations $B$ may be eliminated. One obtains

$$
\begin{aligned}
& A(x)=\frac{\lambda g}{2} \lim _{\xi \rightarrow 0} F^{\prime}(\xi)\left\{: \bar{\psi}(x+\xi) \gamma^{5} \psi(x-\xi):+: \bar{\psi}(x-\xi) \gamma^{5} \psi(x+\xi):\right\} \\
& F(\xi)=\frac{\operatorname{det}\left(a_{i k}(\xi)\right)}{\lambda a_{22}(\xi) \delta m_{1}^{2}(\xi)-g a_{12}(\xi) \delta m_{2}^{2}(\xi)}
\end{aligned}
$$

provided

$$
\begin{aligned}
& \mid \lim _{\xi \rightarrow 0}\left(\delta m_{1}^{2}(\xi)-\frac{g a_{12}(\xi)}{\lambda a_{22}(\xi)} \delta m_{2}^{2}(\xi) \mid=\infty\right. \\
& \mid \lim _{\xi \rightarrow 0}\left(\delta m_{2}^{2}(\xi)-\frac{\lambda a_{22}(\xi)}{g a_{12}(\xi)} \delta m_{1}^{2}(\xi) \mid=\infty .\right.
\end{aligned}
$$

${ }^{6}$ It has also been observed by K. WILson [18] that two independent finite currents can be constructed from $\bar{\psi} \gamma_{5} \psi$ and $A^{3}$. 
Inserting (2.14) into (2.1) one sees that the spinor field satisfies a field equation which formally corresponds to the Lagrangian

$$
\mathfrak{L}=\frac{i}{2}\left(\bar{\psi} \gamma^{\mu} \partial_{\mu} \psi-\partial_{\mu} \bar{\psi} \gamma^{\mu} \psi\right)-M_{0} \bar{\psi} \psi+G_{0}\left(\bar{\psi} \gamma_{5} \psi\right)^{2}
$$

Hence we have a certain equivalence of the pseudoscalar meson-nucleon interaction to a direct Fermi coupling if the stated conditions are satisfied. It should be noted, however, that after elimination of the meson field the model still contains four independent parameters $\lambda, g, M$ and $m$. Since $m$ is arbitrary the meson cannot be interpreted as composite particle in this context. The fact that a four parameter solution $\psi$ may correspond to a two parameter Lagrangian (2.16) merely reflects the ambiguity in quantizing (2.16).

\section{Proportionality of Vector Meson Fields and Corresponding Currents}

We consider a model described by a local, relativistic Lagrangian $\mathfrak{L}$ involving massive vector fields $V_{1}^{\mu}, \ldots, V_{N}^{\mu}$. For the free Lagrangian $\mathfrak{l}_{k}$ of the field $V_{k}^{\mu}$ we choose the Proca-Wentzel form $[19,20]$

$$
\begin{gathered}
\mathfrak{I}_{k}=-\frac{1}{4} V_{k 0 \mu \nu} V_{k 0}^{\mu \nu}+\frac{1}{2} m_{k 0}^{2} V_{k 0 \mu} V_{k 0}^{\mu} \\
V_{k 0 \mu \nu}=\partial_{\mu} V_{k 0 \nu}-\partial_{\nu} V_{k 0 \mu}
\end{gathered}
$$

$\mathfrak{L}_{k}$ is defined as the sum of terms in $\mathfrak{L}$ which are quadratic in $V_{k}^{\mu}$ and its derivatives. The subscript 0 refers to unrenormalized quantities. The field equation of $V_{k}^{\mu}$ then takes the form

where

$$
-\partial_{\nu} V_{k}^{\mu \nu}+m_{k 0}^{2} V_{k}^{\mu}=Z_{k 3}^{-1 / 2} g_{k 0} j_{k}^{\mu}
$$

$$
V_{k}^{\mu}=Z_{3}^{-1 / 2} V_{0 k}^{\mu}
$$

are the renormalized fields.

Since some of the constants $m_{k 0}, g_{k 0}, Z_{k 3}^{-1}$ may be infinite we replace (3.2) by the more meaningful equation

$$
-\partial_{\nu} V_{k}^{\mu \nu}(x)=\lim _{\xi \rightarrow 0}\left(Z_{k 3}^{-1 / 2}(\xi) g_{k 0}(\xi) j_{k}^{\mu}(x \xi)-m_{k 0}^{2}(\xi) V_{k}^{\mu}(x)\right)
$$

with suitably defined currents $j_{k}^{\mu}(x \xi)$ valid for matrix elements

$$
\left(\Phi_{1}, \ldots \Phi_{2}\right)
$$

taken between state vectors from appropriate domains $D_{1}, D_{2}$.

Assuming now that the bare mass associated with the field $V_{k}^{\mu}$ is infinite

$$
\left|\lim _{\xi \rightarrow 0} m_{k 0}^{2}(\xi)\right|=\infty
$$


equation (3.3) implies

$$
V_{k}^{\mu}(x)=\lim _{\xi \rightarrow 0} \frac{g_{k 0}(\xi) j_{k}^{\mu}(x \xi)}{Z_{k 3}^{1 / 2}(\xi) m_{k 0}^{2}(\xi)}
$$

If furthermore the limit

$$
\lim _{\xi \rightarrow 0} j_{k}^{\mu}(x \xi)=j_{k}^{\mu}(x)
$$

exists for matrix elements (3.4) we have the result

$$
V_{k}^{\mu}(x)=\alpha_{k} j_{k}^{\mu}(x), \quad \alpha_{k}=\lim _{\xi \rightarrow 0} \frac{g_{k 0}(\xi)}{Z_{k 3}^{1 / 2}(\xi) m_{k 0}^{2}(\xi)} .
$$

This is a rigorous version of the argument given by KROLL, LeE and ZUMINo that a vector field should be proportional to the corresponding current in case of infinite mass renormalization.

The limit (3.7) formally corresponds to the unrenormalized current $j_{k}^{\mu}$ as defined by (3.2). For a neutral vector meson field coupled to the current of a spin 1/2 field the existence of (3.7) will be shown in section 5 .

It should be remarked that for many models the current $j_{k}^{\mu}$ as defined by the right hand side of (3.2) is different from the conventional current $J_{k}^{\mu}$ which occurs in the minimal coupling to an electromagnetic field. We illustrate this point at the example of an isotopic spin doublet $\psi$ of spin 1/2 fields coupled to a massive Yang-Mills field $V_{k}^{\mu}$. For this model the isotopic spin part of the minimal electromagnetic current is given by the 3-component of

$$
\begin{aligned}
\vec{J}^{\mu} & =\bar{\psi}_{0} \gamma^{\mu} \vec{\tau} \psi_{0}+2 \vec{V}_{0 \nu} \times \vec{W}_{0}^{\nu \mu} \\
\vec{W}_{0}^{\mu \nu} & =\partial^{\mu} \vec{V}_{0}^{\nu}-\partial^{\nu} \vec{V}_{0}^{\mu}+2 g_{0} \vec{V}_{0}^{\mu} \times \vec{V}_{0}^{\nu} .
\end{aligned}
$$

The field equation of the Yang-Mills field, however, is

$$
\partial_{\nu} \vec{W}_{0}^{\mu \nu}+m_{0}^{2} \vec{V}^{\mu}=g_{0} \vec{j}^{\mu}
$$

so that the current defined by (3.2) becomes

$$
\vec{j}^{\mu}=\vec{J}^{\mu}-2 \partial_{\nu}\left(\vec{V}_{0}^{\mu} \times \vec{V}_{0}^{\nu}\right) \text {. }
$$

It is an essential assumption in the concept of field algebra [21, 22] that in the weak and electromagnetic interactions of hadrons the currents $j_{k}^{\mu}$ or fields $V_{k}^{\mu}$ occur rather than the minimal currents $J_{k}^{\mu}$.

\section{Field and Current Commutators in Neutral Vector Meson Theories}

This section deals with equal time commutation relations of fields and currents for neutral vector meson theories. The discussion is formal in so far as we do not attempt to give a precise meaning to equal time commutation relations. 
We consider a neutral vector meson field coupled to the current of a charged spin 1/2 field. For this model JoHNson [16] derived the sum rule

$$
\begin{aligned}
\frac{1}{Z_{3} m_{0}^{2}} & =\frac{1}{m^{2}}+\int_{x_{0}^{2}} \frac{\varrho\left(\varkappa^{2}\right)}{\varkappa^{2}} d \varkappa^{2} \\
0 & <Z_{3} m_{0}^{2} \leqq m^{2}<\varkappa_{0}^{2}
\end{aligned}
$$

where $Z_{3}$ is the wave function renormalization constant of the meson field, $m_{0}$ the bare mass, $\varrho$ the spectral weight function of the propagator. For the derivation Johnson used the Lagrangian of the Proca formulation

$$
\begin{aligned}
\mathfrak{L} & =\mathfrak{L}_{V}+\mathfrak{S}_{\mathrm{int}}+\mathfrak{I}_{\varphi} \\
\mathfrak{L}_{V} & =-\frac{1}{4} V_{0 \mu \nu} V_{0}^{\mu \nu}+\frac{1}{2} m_{0}^{2} V_{0 \mu} V_{0}^{\mu} \\
\mathfrak{L}_{\mathrm{int}} & =-g_{0} j_{\mu} V_{0}^{\mu} \\
\mathfrak{L}_{\varphi} & =\frac{i}{2}\left(\bar{\varphi}_{0} \gamma^{\mu} \partial_{\mu} \varphi_{0}-\partial_{\mu} \bar{\varphi}_{0} \gamma^{\mu} \varphi_{0}\right)-M_{0} \bar{\varphi}_{0} \varphi_{0} \\
V_{0}^{\mu \nu} & =\partial^{\mu} V_{0}^{\nu}-\partial^{\nu} V_{0}^{\mu}, j^{\mu}=\bar{\varphi}_{0} \gamma^{\mu} \varphi_{0} .
\end{aligned}
$$

The renormalized field operators and coupling constant are

$$
V^{\mu}=Z_{3}^{-1 / 2} V_{0}^{\mu}, \varphi=Z_{2}^{-1 / 2} \varphi_{0}, g=Z_{3}^{1 / 2} g_{0} .
$$

The independent pairs of canonically conjugate fields are

$$
\left(V^{k}, V^{0}\right) \quad k=1,2,3 .
$$

In order to obtain the commutators involving $V^{0}$ one solves the field equation formally by

and assumes

$$
V^{0}=\frac{g j^{0}}{Z_{3} m_{0}^{2}}+\frac{1}{m_{0}^{2}} \sum_{j=1}^{3} \partial_{j} V^{0 j}
$$

$$
\left[j^{0}(x) V^{k}(y)\right]=0 \text { for } x^{0}=y^{0}, k=1,2,3 .
$$

(4.4) is the basic hypothesis in JoHNson's work which yields the equal time commutator of $V^{k}, V^{0}$. The relation

$$
\left[V^{k}(x), V^{0}(y)\right]=\frac{i}{Z_{3} m_{0}^{2}} \partial^{k} \delta_{3}(x-y)
$$

leads to the sum rule (4.1) provided the meson propagator has an unsubtracted integral representation?.

${ }^{7}$ It is assumed here that $m^{2}$ is the only square mass eigenvalue of spin 1 in the interval $0 \leqq K^{2}<K_{0}^{2}$. 
We will now show that assumption (4.4) is inconsistent if the bare mass of the meson is infinite. We write the field equation in the limit form (3.3) and obtain with $g_{0}(\xi)=Z_{3}^{1 / 2}(\xi) g$

provided

$$
V^{\mu}(x)=g \lim _{\xi \rightarrow 0} \frac{j^{\mu}(x \xi)}{Z_{3}(\xi) m_{0}^{2}(\xi)}
$$

$$
\lim _{\xi \rightarrow 0} m_{0}^{2}(\xi)=\infty \text {. }
$$

According to the sum rule (4.1) the limit

$$
\bar{m}^{2}=\lim _{\xi \rightarrow 0} Z_{3}(\xi) m_{0}^{2}(\xi)
$$

exists and is different from zero. Hence the limit of the current operator exists and is related to the field operator by ${ }^{8}$

$$
V^{\mu}(x)=\frac{g}{\bar{m}^{2}} j^{\mu}(x), j^{\mu}(x)=\lim _{\xi \rightarrow 0} j^{\mu}(x, \xi) .
$$

With this result, however, the commutation relations (4.4) and (4.5) are incompatible.

The source of the trouble is of course the formal use of the field equation in (4.3) which is not permitted for divergent constants $m_{0}, g_{0}$. Indeed, in this case the corrected version of (4.3) is precisely

$$
V^{0}(x)=\frac{g}{\bar{m}^{2}} j^{0}(x) \text {. }
$$

Hence any assumption on the commutator $\left[j^{0}, V^{k}\right]$ would anticipate the commutation relations of $V^{0}$ and $V^{k}$.

The conclusion is that the canonical quantization rules of the ProcaWentzel formulation do not provide sufficient information for proving JoHNson's sum rule. On the other hand this sum rule has been derived by SYMANZIK [17] by quantizing first the Lagrangian (5.2) of the following section. Defining then the Proca field $V^{\mu}$ by the gauge transformation (5.12) the equal time commutation relations of $V^{\mu}, \hat{V}^{v}$ are obtained including (4.5). Relation (4.5) implies JoHnson's sum rule (4.1) provided the meson propagator satisfies an unsubtracted integral representation.

The equal time commutators of $V^{\mu}$ combined with (4.8) yield the current-field commutators as well as the commutators of the current components

$$
\begin{aligned}
{\left[V^{k}(x) j^{0}(y)\right] } & =\frac{i}{g} \partial^{k} \delta_{3}(x-y) \quad x^{0}=y^{0} \\
{\left[j^{0}(x) j^{0}(y)\right] } & =\left[j^{k}(x) j^{l}(y)\right]=0 \quad k, l=1,2,3 \\
{\left[j^{k}(x) j^{0}(y)\right] } & =i \frac{\bar{m}^{2}}{g^{2}} \partial^{k} \delta_{3}(x-y)
\end{aligned}
$$

${ }^{8}$ For a more detailed discussion of this argument see Section 5. 
provided

$$
\lim _{\xi \rightarrow 0} m_{0}^{2}(\xi)=\infty \text {. }
$$

Equ. (4.11) is in agreement with Schwinger's results [23]

$$
\left\langle\left[j^{k}(x) j^{0}(y)\right]\right\rangle_{0}=i c \partial^{k} \delta_{3}(x-y) .
$$

It should be remarked that the commutators (4.9-11) cannot be computed from the corresponding commutators of $j^{\mu}(x \xi)$ by taking the limit $\xi \rightarrow 0$. As was pointed out in ref. [11] the relation

does not imply

$$
j^{\mu}(x)=\lim _{\xi \rightarrow 0} j^{\mu}(x \xi)
$$

or

$$
\begin{aligned}
{\left[j^{k}(x) V^{0}(y)\right] } & =\lim _{\xi \rightarrow 0}\left[j^{k}(x \xi) V^{0}(y)\right] \\
x^{0} & =y^{0} \\
{\left[j^{\mu}(x) j^{\nu}(y)\right] } & =\lim _{\xi, \eta \rightarrow 0}\left[j^{\mu}(x \xi) j^{\nu}(y y)\right] .
\end{aligned}
$$

The reason why (4.14) fails to follow is that (4.12) converges in the weak limit only. (4.13) is not implied since the validity of (4.12) is restricted to matrix elements (3.4) for which the field equation (3.3) is correct. For instance, in order to derive the vacuum expectation value of (4.13) one would need the field equation for matrix elements

$$
\begin{gathered}
(\Omega, \ldots \Phi) \quad \text { and } \quad(\Phi, \ldots \Omega) \\
\Phi=\int d_{3} y V^{0}(y) f(\vec{y}) \Omega .
\end{gathered}
$$

But for divergent $Z_{2}^{-1}$ or $m_{0}$ the left hand side $\delta_{v} V^{0 v}(x)$ of the field equation is not even defined for such matrix elements.

In conclusion we remark that JoHNson's hypothesis (4.4) is correct for a finite theory $\left(m_{0} \neq \infty\right)$ and can be derived from the canonical commutation rules of the Lagrangian (5.2).

\section{The Current Operator in Neutral Vector Meson Theories}

In this section we propose an explicit form of the current operator $j^{\mu}(x \xi)$ for the model of a neutral vector meson field coupled to the current of a charged spin $1 / 2$ field. It will further be shown that the limit

$$
j^{\mu}(x)=\lim _{\xi \rightarrow 0} j^{\mu}(x \xi)
$$

should exist in a consistent theory. The model has the advantage that it can be renormalized by conventional methods ${ }^{9}$. So perturbation theory may be used as a guide for finding a suitable definition of $j^{\mu}(x \xi)$. Using the Gupta-Bleuler metric the renormalization program has been

${ }^{\circ}$ So far this has not been possible for Yang-Mills type interactions unless an infinite number of counterterms is admitted. 
carried out in detail by SYMANZIK [17] on the basis of the Lagrangian ${ }^{10,11}$

$$
\begin{aligned}
\mathfrak{L} & =\mathfrak{I}_{A}+\mathfrak{L}_{\mathrm{int}}+\mathfrak{I}_{\varphi} \\
\mathfrak{I}_{A} & =-\frac{1}{4} A_{0 \mu \nu} A_{0}^{\mu \nu}+\frac{1}{2} m_{0}^{2} A_{0 \mu} A_{0}^{\mu}-\frac{1}{2} \frac{m_{0}^{2}}{\lambda^{2}}\left(\partial_{\mu} A_{0}^{\mu}\right)^{2} \\
\mathfrak{L}_{\text {int }} & =-g_{0} \hat{\jmath}_{\mu} A_{0}^{\mu} \\
\mathfrak{I}_{\psi} & =\frac{i}{2}\left(\bar{\psi}_{0} \gamma^{\mu} \partial_{\mu} \psi_{0}-\partial_{\mu} \bar{\psi}_{0} \gamma^{\mu} \psi_{0}\right)-M_{0} \bar{\psi}_{0} \psi_{0} \\
A_{0}^{\mu \nu} & =\partial^{\mu} A_{0}^{v}-\partial^{\nu} A_{0}^{\mu}, \hat{\jmath}^{\mu}=\bar{\psi}_{0} \gamma^{\mu} \psi_{0} .
\end{aligned}
$$

The subscript $t_{0}$ refers to unrenormalized quantities. The renormalized field operators and coupling constant are

$$
A^{\mu}=Z_{3}^{-1 / 2} A_{0}^{\mu}, \psi=Z_{2}^{-1 / 2} \psi_{0}, g=Z_{3}^{1 / 2} g_{0} .
$$

As a finite form of the meson field equation we propose

$$
\begin{gathered}
-\partial_{\nu} A^{\mu \nu}(x)=\lim _{\xi \rightarrow 0}\left\{g Z_{3}^{-1}(\xi) \hat{\jmath}^{\mu}(x \xi)-m_{0}^{2}(\xi) A^{\mu}(x)+\frac{m_{0}^{2}(\xi)}{\lambda^{2}} \partial^{\mu} \partial_{\nu} A^{\nu}(x)\right\} \\
\left(\square+\lambda^{2}\right) \partial_{\nu} A^{\nu}(x)=0,
\end{gathered}
$$

for matrix elements between suitable state vectors. $\lambda$ is an arbitrary finite and positive parameter which may be identified with the renormalized mass ${ }^{12}$. For the current operator we propose

$$
\begin{aligned}
\hat{\jmath}^{\mu}(x \xi) & =Z_{2}^{\prime}(\xi) \lim _{\xi \rightarrow \xi} \frac{\hat{\jmath}^{\mu}(x \xi \eta)}{Y^{\prime}(\xi \eta)} \\
\hat{\jmath}^{\mu}(x \xi \eta) & =\frac{1}{2}\left(\hat{Q}^{\mu}(x \xi \eta)+\hat{Q}^{\mu}(x,-\xi,-\eta)\right) \\
\hat{Q}^{\mu}(x \xi \eta) & =: \bar{\psi}(x+\xi) \gamma^{\mu} e^{-i g \int_{x-\eta}^{x+\eta} d y^{\mu} A_{\mu}(y)} \psi(x-\xi):
\end{aligned}
$$

10 For a discussion of (5.2) see, for instance, ref. [24], section 5 and ref. [25], section 3.

11 Lagrangian (5.2) is closely related to the Lagrangian of the STUECKELBERG [26] formalism

$$
\begin{aligned}
& \mathfrak{L}_{A B}=-\frac{1}{4} A_{0 \mu \nu} A_{0}^{\mu \nu}+\frac{1}{2} m_{0}^{2} U_{0 \mu} U_{0}^{\mu}-\frac{1}{2} \frac{m_{0}^{2}}{\lambda^{2}} \chi_{0}^{2} \\
& U_{0}^{\mu}=A_{0}^{\mu}-\frac{1}{m_{0}} \partial_{\mu} B, \chi_{0}=\partial^{\mu} A_{0 \mu}+\frac{\lambda^{2}}{m_{0}} B
\end{aligned}
$$

which is invariant under gauge transformations

$$
A_{0 \mu}^{\prime}=A_{0 \mu}+\frac{1}{m_{0}} \partial_{\mu} \Lambda, B^{\prime}=B+\Lambda
$$

Up to a divergence (*) equals $\mathfrak{L}_{A}+\mathfrak{L}_{B}$ with $\mathfrak{L}_{B}$ denoting the free Lagrangian of a scalar field $B$ of mass $\lambda$. Apart from a minor modification (*) was proposed by FuJII and KAMEFUCHI [27].

12 In perturbation theory where $m_{0}=\infty$ it would be inconsistent to set $\lambda=m_{0}$ because (5.4) holds for the renormalized field. 
This form is suggested by the work of VALATIN [4] and BRANDT [7] in quantum electrodynamics ${ }^{13} \cdot x \pm \eta$ are points on the straight line connecting $x-\xi$ with $x+\xi$. The integral is extended along this line.

$::$ denotes the generalized normal product defined recursively by

$$
\mathfrak{O}_{1} \ldots \mathfrak{O}_{n}=: \mathfrak{O}_{1} \ldots \mathfrak{O}_{n}:+\Sigma\left\langle\mathfrak{O}_{i 1} \ldots \mathfrak{O}_{i_{\alpha}}\right\rangle_{\mathfrak{0}}: \mathfrak{O}_{i_{\alpha+1}} \ldots \mathfrak{O}_{i n}:
$$

for field operators $A_{\mu}$ or $\psi$ at spacelike relative distances ${ }^{14}$. The sum extends over the subsets $\left(i_{1}, \ldots, i_{\alpha}\right)$ of $(1, \ldots, n)$.

$$
i_{1}<\ldots<i_{\alpha}, i_{\alpha+1}<\ldots<i_{n} .
$$

In perturbation theory (5.5-7) may presumably be replaced by the simpler expression

$$
\begin{aligned}
& \hat{\jmath}^{\mu}(x \xi)=Z_{2}^{\prime}(\xi) \frac{1}{2}\left(\hat{Q}^{\mu}(x \xi)+\hat{Q}^{\mu}(x,-\xi)\right) \\
& \hat{Q}^{\mu}(x \xi)= \bar{\psi}(x+\xi) \gamma^{\mu} \psi(x-\xi) \\
&-\left\langle\bar{\psi}(x+\xi) \gamma^{\mu} \psi(x-\xi)\right\rangle_{0} \sum_{n=1}^{N} \frac{(-i g)^{n}}{n !}:\left[\int_{x-\xi}^{x+\xi} d y^{\mu} A_{\mu}(y)\right]^{n}:
\end{aligned}
$$

where only a few terms in the expansion of the exponential are needed.

Using the methods of ref. [4-9] it should not be difficult to check the validity of (5.3-9) in renormalized perturbation theory.

For the limit form of the Dirac equation see the Appendix, equ. (A. 4). Since the divergence $\partial_{\mu} A^{\mu}$ is a solution of the Klein-Gordon equation (5.4) the commutators $\left[\partial_{\nu} A^{\nu}, \psi\right],\left[\partial_{\nu} A^{\nu}, A^{\mu}\right]$ must be well-defined solutions of the Klein-Gordon equation in a consistent theory. As is dicussed in the Appendix this leads to the requirement that

$$
\bar{m}^{2}=\lim _{\xi \rightarrow 0} Z_{3}(\xi) m_{0}^{2}(\xi) \neq 0, \infty .
$$

In this argument it is not used that the meson propagator satisfies an unsubtracted integral representation.

We will now derive the meson field equation in the Proca formulation. Let $p$ be the projection operator on the subspace of the physical state vectors defined by

$$
\left(\partial_{\mu} A^{\mu}(x)\right)^{+} \Phi=0 .
$$

+ denotes the positive frequency part. The meson field operator of the Proca formulation is then introduced by

$$
V^{\mu}(x)=\hat{\nabla}^{\mu}(x) p, \quad \hat{\nabla}^{\mu}(x)=A^{\mu}(x)+\frac{1}{\lambda^{2}} \partial^{\mu} \partial^{\nu} A_{v}(x) .
$$

The definition of the Fermi field operator $\varphi$ in the Proca formulation is given in the Appendix. For $V^{\mu}(s)$ one obtains from (5.3) the field equation in Proca form

$-\partial_{\nu} V^{\mu \nu}(x)=\lim _{\xi \rightarrow 0}\left\{g Z_{3}^{-1}(\xi) j^{\mu}(x \xi)-m_{0}^{2}(\xi) V^{\mu}(x)\right\}, \quad \partial_{\mu} V^{\mu}(x)=0$

18 For the motivation of the exponential in (5.7) see for instance ref. [28].

14 This presciption should be applied to each term of (5.7) after expansion of the exponential. 
with

$$
j^{\mu}(x \xi)=\hat{\jmath}^{\mu}(x \xi) p .
$$

As is shown in the Appendix the current operator $j^{\mu}(x \xi)$ may be expressed in terms of the Proca field by

$$
\begin{gathered}
j^{\mu}(x \xi)=Z_{2}(\xi) \lim _{\eta \rightarrow \xi} \frac{j^{\mu}(x \xi \eta)}{Y(\xi \eta)} \\
j^{\mu}(x \xi \eta)=\frac{1}{2}\left(Q^{\mu}(x \xi \eta)+Q^{\mu}(x,-\xi,-\eta)\right) \\
Q^{\mu}(x \xi \eta)=: \bar{\varphi}(x+\xi) \gamma^{\mu} e^{-i g{ }_{x-\eta}^{x+\eta} d y^{\mu} \nabla_{\mu}(y)} \varphi(x-\xi):
\end{gathered}
$$

Finally, we derive the existence of the limit (5.1). If

(5.10) and (5.13) imply

$$
\lim _{\xi \rightarrow 0} m_{0}^{2}(\xi)=\infty
$$

$$
\lim _{\xi \rightarrow 0} j^{\mu}(x \xi)=\frac{\bar{m}^{2}}{g} V^{\mu}(x)^{15}
$$

for all matrix elements for which (5.13) is valid. For these matrix elements the existence of the limit (5.1) follows with the current defined by

$$
j^{\mu}(x)=\frac{\bar{m}^{2}}{g} V^{\mu}(x)
$$

By definition $j^{\mu}(x)$ is an operator valued distribution with the same domain as $V^{\mu}(x)$.

If $\lim _{\xi \rightarrow 0} m_{0}^{2}(\xi)=m_{0}^{2} \neq 0$ equ. (5.1) follows similarly with the current defined by

$$
j^{\mu}(x)=\frac{\bar{m}^{2}}{g m_{0}^{2}}\left(-\partial_{\nu} V^{\mu \nu}(x) m_{0}^{2} V^{\mu}(x)\right)
$$

\section{Impossibility of Coupling Independent Vector Meson Fields to the Same Current}

We consider two vector meson fields $V_{1}^{\mu}$ and $V_{2}^{\mu}$ in the Proca-Wentzel formulation which are coupled to the same current. It will be shown, that $V_{1}^{\mu}$ and $V_{2}^{\mu}$ must be proportional provided their bare masses are both infinite. We give two alternative formulations ${ }^{16}$ of the hypothesis that $V_{1}^{\mu}$ and $V_{2}^{\mu}$ are coupled to the same current.

15 (5.19) holds in perturpation theory up to an expression of the form $\lim _{\xi \rightarrow 0} Z_{3}(\xi) \partial_{\nu} V^{\mu \nu}(x)$ which should be considered to be zero.

16 These formulations correspond to a free meson Lagrangian

and an interaction term

$$
\text { (a) } \mathscr{L}_{0}=-\frac{1}{4} \sum_{k=1}^{2} V_{k 0 \mu \nu} V_{k 0}^{\mu \nu}+\frac{1}{2} \sum_{k=1}^{2} m_{0 k}^{2} V_{k 0 \mu} V_{k 0}^{\mu}
$$

$$
\text { (b) } \mathscr{L}_{\text {int }}=-\sum_{k=1} g_{0 k} j_{\mu} V_{k 0}^{\mu} \text {. }
$$

One can of course introduce mixing terms of the form $V_{1 \mu \nu} V_{2}^{\mu \nu}, V_{1 \mu} V_{2}^{\mu}$ in $\mathscr{L}_{0}$. It can be shown that the mixing matrices must be made positive lest ghost states appear in the theory. But then the meson Lagrangian can be brought into the form (a) by a linear transformation of the field (see ref. [13]). 
(i) The fields $V_{1}^{\mu}, V_{2}^{\mu}$ satisfy the field equations

$$
-\partial_{\nu} V_{k}^{\mu \nu}(x)=\lim _{\xi \rightarrow 0}\left(f_{k}(\xi) j^{\mu}(x \xi)-m_{k 0}^{2}(\xi) V_{k}^{\mu}(x) .\right.
$$

valid for matrix elements

$$
\left(\Phi_{1}, \ldots \Phi_{2}\right) \quad \Phi_{1} \in D_{1}, \Phi_{2} \in D_{2}
$$

with suitable domains $D_{1}, D_{2}$.

In this case we do not assume that the limit $\lim _{\xi \rightarrow 0} j^{\mu}(x \xi)$ exists.

(ii) The fields $V_{1}^{\mu}, V_{2}^{\mu}$ satisfy the field equations

$$
-\partial_{\nu} V_{k}^{\mu \nu}(x)=\lim _{\xi \rightarrow 0}\left(f_{k}(\xi) j_{k}^{\mu}(x \xi)-m_{k 0}^{2}(\xi) V_{k}^{\mu}(x)\right)
$$

valid for matrix elements (6.2). Here the currents may have a different $\xi$-dependence, but it is assumed that they approach the same nonvanishing limit

$$
\lim _{\xi \rightarrow 0}\left(\Phi_{1}, j_{1}^{\mu}(x \xi) \Phi_{2}\right)=\lim _{\xi \rightarrow 0}\left(\Phi_{1}, j_{2}^{\mu}(x \xi) \Phi_{2}\right) \neq 0
$$

for matrix elements (6.2).

In both cases it will be shown that $V_{1}^{\mu}$ and $V_{2}^{\mu}$ are proportional provided

$$
\left|\lim _{\xi \rightarrow 0} m_{k 0}^{2}(\xi)\right|=\infty . \quad k=1,2
$$

We begin with case (i). Equ. (6.1) is equivalent to

$$
\begin{gathered}
V_{k}^{\mu}(x)=h_{k}(\xi) j^{\mu}(x \xi)+p_{k}^{\mu}(x \xi) \\
h_{k}(\xi)=\frac{f_{k}(\xi)}{m_{k_{0}}^{2}(\xi)}, p_{k}^{\mu}(x \xi)=\frac{o_{k}^{\mu}(x \xi)+\partial_{\nu} V_{k}^{\mu \nu}(x)}{m_{k_{0}}^{2}(\xi)}
\end{gathered}
$$

with

The hypothesis

$$
\lim _{\xi \rightarrow 0} o_{k}^{\mu}(x \xi)=0 .
$$

$$
\left|\lim _{\xi \rightarrow 0} m_{k_{0}}^{2}(\xi)\right|=\infty
$$

implies

$$
\lim _{\xi \rightarrow 0} p_{k}^{\mu}(x \xi)=0 .
$$

Let $\xi_{n}$ be an arbitrary sequence of spacelike points approaching the origin. We choose a subsequence $\xi_{n}^{\prime}$ such that the sequence

$$
\frac{h_{1}\left(\xi_{n}^{\prime}\right)}{h_{2}\left(\xi_{n}^{\prime}\right)}
$$

has only one accumulation point. We assume that

$$
h=\lim _{n \rightarrow \infty} \frac{h_{1}\left(\xi_{n}^{\prime}\right)}{h_{2}\left(\xi_{n}^{\prime}\right)}
$$

is finite. This is no loss of generality since

$$
\lim _{n \rightarrow \infty} \frac{h_{1}\left(\xi_{n}^{\prime}\right)}{h_{2}\left(\xi_{n}^{\prime}\right)}=\infty
$$


can be reduced to (6.9) by interchanging $V_{1}^{\mu}$ and $V_{2}^{\mu}$. Multiplying (6.6) for $k=2$ by $\frac{h_{1}}{h_{2}}$ and taking the limit $n \rightarrow \infty$ we obtain

Hence

$$
\lim _{n \rightarrow \infty} \frac{h_{1}\left(\xi_{n}^{\prime}\right)}{h_{2}\left(\xi_{n}^{\prime}\right)} V_{2}^{\mu}(x)=\lim _{n \rightarrow \infty} h_{1}\left(\xi_{n}^{\prime}\right) j^{\mu}\left(x \xi_{n}^{\prime}\right)=V_{1}^{\mu}(x)
$$

and

$$
h=\lim _{n \rightarrow \infty} \frac{h_{1}\left(\xi_{n}^{\prime}\right)}{h_{2}\left(\xi_{n}^{\prime}\right)} \neq 0, \infty
$$

$$
V_{1}^{\mu}=h V_{2}^{\mu}
$$

In case (ii) the field equation (6.3) is equivalent to

with

$$
V_{k}^{\mu}(x)=h_{k}(\xi) j_{k}^{\mu}(x \xi)+p_{k}^{\mu}(x \xi)
$$

If

$$
\lim _{\xi \rightarrow 0} o_{k}^{\mu}(x \xi)=0 \text {. }
$$

we get

$$
\left|\lim _{\xi \rightarrow 0} m_{k 0}^{2}(\xi)\right|=\infty
$$

It follows that

$$
\left(\Phi_{1}, V_{k}^{\mu}(x) \Phi_{2}\right)=\lim _{\xi \rightarrow 0} h_{k}(\xi)\left(\Phi_{1}, j_{k}^{\mu}(x \xi) \Phi_{2}\right)
$$

$$
h_{k}=\lim _{\xi \rightarrow 0} h_{k}(\xi) \neq 0, \infty
$$

exists and does not vanish. Hence

$$
V_{1}^{\mu}=\frac{h_{1}}{h_{2}} V_{2}^{\mu}
$$

In case (i) we can further show that

$$
\lim _{\xi \rightarrow 0} \frac{m_{10}^{2}(\xi)}{m_{20}^{2}(\xi)}=1
$$

i.e. the bare masses of the two fields are equal. This result is an application of the following uniqueness theorem.

Let $V^{\mu}$ be a solution of the field equation

Then the equation

$$
-\partial_{\nu} V^{\mu \nu}(x)=\lim _{\xi \rightarrow 0}\left(f(\xi) j^{\mu}(x \xi)-m_{0}^{2}(\xi) V^{\mu}(x)\right) .
$$

$$
-\partial_{\nu} V^{\mu \nu}(x)=\lim _{\xi \rightarrow 0}\left(f^{\prime}(\xi) j^{\mu}(x \xi)-m_{0}^{2}(\xi) V^{\mu}(x)\right)
$$

is satisfied by $f^{\prime}$ and $m_{0}^{\prime}$ for the same matrix elements if and only if

$$
\begin{aligned}
\lim _{\xi \rightarrow 0} \frac{f^{\prime}(\xi)}{f(\xi)} & =1 \\
\lim _{\xi \rightarrow 0}\left(m_{0}^{2}(\xi) \frac{f^{\prime}(\xi)}{f(\xi)}-m_{0}^{\prime 2}(\xi)\right) & =0 .
\end{aligned}
$$


Relation (6.18) implies

$$
\lim _{\xi \rightarrow 0} \frac{m_{0}^{2}(\xi)}{m_{0}^{2}(\xi)}=1
$$

The sufficiency of $(6.18)$ is obvious. In order to prove that (6.18) is necessary we assume both equations (6.16) and (6.17) to be valid. Then we have

with

$$
-\partial_{\nu} V^{\mu \nu}(x)+m_{0 k}^{2}(\xi) V^{\mu}(x)=f_{k}(\xi) j^{\mu}(x \xi)+o_{k}^{\mu}(x \xi)
$$

$$
\lim _{\xi \rightarrow 0} o_{k}^{\mu}(x, \xi)=0
$$

Let $\xi_{n}$ be a sequence with

$$
\xi_{n}^{2}<0, \quad \lim _{\xi \rightarrow 0} \xi_{n}=0, \quad\left|\xi^{\mu} / \sqrt{-\xi^{2}}\right| \leqq B .
$$

To every accumulation point $f$ of the sequence $\frac{f_{2}\left(\xi_{n}\right)}{f_{1}\left(\xi_{n}\right)}$ we choose a subsequence $\xi_{n}^{\prime}$ of $\xi_{n}$ with

We first assume

$$
\lim _{n \rightarrow \infty} \frac{f_{2}\left(\xi_{n}^{\prime}\right)}{f_{1}\left(\xi_{n}^{\prime}\right)}=f
$$

Form (6.20) we obtain

$$
|f| \leqq 1
$$

The limit

$$
\left(\frac{f_{2}}{f_{1}}-1\right)\left(-\partial_{\nu} V^{\mu \nu}\right)+\left(m_{01}^{2} \frac{f_{2}}{f_{1}}-m_{02}^{2}\right) \nabla^{\mu}=\frac{f_{2}}{f_{1}} o_{1}^{\mu}-o_{2}^{\mu} .
$$

$$
t=\lim _{n \rightarrow \infty}\left(m_{01}^{2}\left(\xi_{n}^{\prime}\right) \frac{f_{2}\left(\xi_{n}^{\prime}\right)}{f_{1}\left(\xi_{n}^{\prime}\right)}-m_{02}^{2}\left(\xi_{n}^{\prime}\right)\right)
$$

exists because of (6.21), (6.23) and (6.24). So we obtain

$$
(f-1)\left(-\partial_{\nu} V^{\mu \nu}(x)\right)+t V^{\mu}(x)=0 \text {. }
$$

Excluding the trivial case that $V^{\mu}$ statisfies a linear equation we get

$$
f=1, \quad t=0 \text {. }
$$

For $|f| \geqq 1$ we obtain in a similar way

$$
\lim _{n \rightarrow \infty} \frac{f_{1}\left(\xi_{n}^{\prime}\right)}{f_{2}\left(\xi_{n}^{\prime}\right)}=1
$$

which is included in (6.23). Since (6.25) holds for every accumulation point (6.22) condition (6.18) follows.

Finally we apply the uniqueness theorem to case (i). According to (6.1) and (6.14) the field $V_{1}^{\mu}$ satisfies both equations

$$
\begin{aligned}
& -\partial_{\nu} V_{1}^{\mu \nu}(x)=\lim _{\xi \rightarrow 0}\left(f_{1}(\xi) j^{\mu}(x \xi)-m_{10}^{2}(\xi) V_{1}^{\mu}(x)\right) \\
& -\partial_{\nu} V_{1}^{\mu \nu}(x)=\lim _{\xi \rightarrow 0}\left(\frac{h_{1}}{h_{2}} f_{1}(\xi) j^{\mu}(x \xi)-m_{20}^{2}(\xi) V_{1}^{\mu}(x)\right) .
\end{aligned}
$$

Hence we obtain (6.15) as a necessary condition. 
Appendix. Limit Form of Local Field Equations for a Neutral Vector Meson Theory

(a) Formulation with Indefinite Metric

We consider the model of a vector meson field coupled to the current of a charged spin 1/2 field. The Lagrangian (5.2) formally leads to the following field equations of the renormalized fields

$$
\begin{gathered}
-\partial_{\nu} A^{\mu \nu}+m_{0}^{2} A^{\mu}+\frac{m_{0}^{2}}{\lambda^{2}} \partial^{\mu} \partial_{\nu} A^{\nu}=g Z_{3}^{-1} \hat{\jmath}^{\mu} \\
\left(\square+\lambda^{2}\right) \partial_{\mu} A^{\mu}=0 \\
i \gamma^{\mu} \partial_{\mu} \psi-M_{0} \psi=g \gamma^{\mu} A_{\mu} \psi .
\end{gathered}
$$

We list some of the commutation relations which follow from the canonical rules and (A.1)

$$
\begin{gathered}
{\left[\partial_{\nu} A^{\nu}(x), \psi(y)\right]=\frac{\lambda^{2} g}{Z_{3} m_{0}^{2}} \psi(y) \Delta_{\lambda}(x-y)} \\
{\left[\partial_{\nu} A^{\nu}(x), A^{\mu}(y)\right]=-\frac{\lambda^{2}}{Z_{3} m_{0}^{2}} i \partial^{\mu} \Delta_{\lambda}(x-y) .}
\end{gathered}
$$

We now propose a finite formulation of the field equations (A.1) and the commutation relations (A.2-3). The field operators are supposed to be operator-valued distributions on a domain $\hat{D}$ which is dense in a Hilbert space $\hat{\mathfrak{Y}}$ of indefinite metric. Except for positive definiteness of the metric the usual postulates of quantum field theory are assumed in the form proposed by JAFFE [3].

As local field equations we propose (5.3-7) and

$i \gamma^{\mu} \partial_{\mu} \psi(x)=\lim _{\xi \rightarrow 0}\left(g \frac{\gamma^{\mu}}{2}\left(A_{\mu}(x+\xi)+A_{\mu}(x-\xi)\right) \psi(x)+M_{0}(\xi) \psi(x)\right)$

to hold for matrix elements.

$$
\left(\Phi_{1}, \ldots \Phi_{2}\right) \quad \Phi_{1} \in \hat{D}_{1}, \Phi_{2} \in \hat{D}_{2} .
$$

$\hat{D}_{1}$ and $\hat{D}_{2}$ are dense linear subsets of $\hat{D}$ with the property that

$$
\begin{aligned}
& \left(\Phi_{1}, A^{\mu}(x) \Phi_{2}\right),\left(\Phi_{1}, \hat{\jmath}^{\mu}(x \xi \eta) \Phi_{2}\right) \\
& \left(\Phi_{1}, A^{\mu}(x+\xi) \psi(x) \Phi_{2}\right),\left(\Phi_{1}, \psi(x) \Phi_{2}\right)
\end{aligned}
$$

are ordinary functions of $x, \xi$ and $\eta$ for

$$
\Phi_{i} \in \hat{D}_{i}, \xi^{2}<0, \quad\left|\xi^{\mu}\right| \leqq \varepsilon^{\mu}>0
$$

and $\eta$ lying on the straight line connecting $-\xi$ with $+\xi^{17}$. Since

$$
A(x)=\frac{1}{\lambda} \partial_{\nu} A^{\nu}(x)
$$

is a solution of the Klein-Gordon equation (5.4) the commutators of $A(x)$

${ }^{17} \varepsilon^{\mu}$ is chosen small enough that the matrix element $\left(\Phi_{1}, \ldots \Phi_{2}\right)$ of (5.7) converges after expansion of the exponential.

$6^{*}$ 
with $\psi(y)$ and $A^{\mu}(y)$ must be well-defined solutions of the Klein-Gordon equation (5.4) to the mass $\lambda$. Hence for the theory to be meaningful the limit

$$
\bar{m}^{2}=\lim _{\xi \rightarrow 0} Z_{3}(\xi) m_{0}^{2}(\xi) \neq 0, \infty
$$

must exist and be different from zero ${ }^{18}$. Accordingly we postulate the commutation relations

$$
\begin{gathered}
{[A(x) \psi(y)]=\frac{\lambda g}{\bar{m}^{2}} \psi(y) \Delta_{\lambda}(x-y)} \\
{\left[A(x) A^{\mu}(y)\right]=-\frac{\lambda}{\bar{m}^{2}} i \partial^{\mu} \Delta_{\lambda}(x-y) .}
\end{gathered}
$$

The last relation implies

$$
[A(x) A(y)]=-i \frac{\lambda^{2}}{\overline{\bar{m}}^{2}} \Delta_{\lambda}(x-y) .
$$

Let $\mathfrak{S}^{\prime}$ be the closure of the subspace which is generated by applying polynomials of creation operators

$$
A_{\bar{f}}^{-}=\int f(x) A^{-}(x) d x
$$

to the vacuum $\Omega . A^{-}(x)$ is the negative frequency part of the free field $A(x), f(x)$ is a suitable test function. $\mathfrak{H}^{\prime}$ is spanned by the vectors

$$
\Phi^{\prime}=A_{\bar{f}_{1}}^{-} \ldots A_{\bar{f}_{n}}^{-} \Omega .
$$

Since the commutator (A.10) has the negative sign an indefinite GuptaBleuler metric must be used in $\mathfrak{G}^{\prime}$. by

Let $\mathfrak{S}$ be the closure of the subspace of physical state vectors defined

$$
A_{f}^{+} \Phi=0, \Phi \in \hat{D} .
$$

$p$ denotes the projection operator on $\mathfrak{G}$. In $\mathfrak{G}$ a positive definite metric is used. The intersections of $\hat{D}, \hat{D}_{1}, \hat{D}_{2}$ with $\mathfrak{G}$ are assumed to be dense in $\mathfrak{G}$ and invariant under $p$

$$
p \hat{D} \leqq \hat{D}, p \hat{D}_{j} \leqq \hat{D}_{j} .
$$

The product $\Phi \times \Phi^{\prime}$ of a physical state vector $\Phi$ and a vector $\Phi^{\prime}$ of the form (A.11) is defined by

$$
\Phi \times \Phi^{\prime}=A_{f_{1}}^{-} \ldots A_{f_{n}}^{-} \Phi, \quad \Phi \in \hat{D} \cap \mathfrak{G} .
$$

The linear span of all vectors (A.11) is dense in $\hat{\mathfrak{Y}}$ and contained in $\hat{D}$. From (A.10), (A.12) it follows

$$
\left(\Phi_{1} \times \Phi_{1}^{\prime}, \Phi_{2} \times \Phi_{2}^{\prime}\right)=\left(\Phi_{1}, \Phi_{2}\right)\left(\Phi_{1}^{\prime}, \Phi_{2}^{\prime}\right) .
$$

(b) Transformation the to Proca-Wentzel Formulation

The fields $V^{\mu}$ and $\varphi$ are defined by (5.12) and

$$
\hat{\varphi}(x)=e^{i \frac{g^{2}}{\bar{m}^{2}} \Delta_{\lambda}^{+}(0)}: e^{-i \frac{g}{\lambda} A(x)}: \psi(x) .
$$

${ }^{18}$ For $\bar{m}=\infty$ the operator $A(x)$ would commute with $\bar{\psi}(y), \psi(y), A_{\mu}(y), A(y)$ at all times. In this case cyclicity of the vacuum state would imply $A(x) \equiv 0$. 
The formal properties of these operator gauge transformations were studied in detail by SymanzIK [17].

Since $\Delta_{\lambda}^{+}$diverges at the origin (A.16) is not well defined. Therefore, we replace (A.16) by the weak limit

$$
\hat{\varphi}(x)=\lim _{\xi \rightarrow 0} e^{i \frac{g^{2}}{\bar{m}^{2}} \Delta_{\lambda}^{+}(\xi)}: e^{-i \frac{g}{\lambda} A(x+\xi)}: \psi(x)
$$

and demonstrate the existence of the limit. (A.17) is supposed to hold for matrix elements of the form (A.14). For a free field $A(z)$ JAFFE [3] has shown that

$$
: e^{-i \frac{g}{\lambda} A(z)}:
$$

is a well defined operator-valued distribution in his formulation of quantum field theory. We study now the properties of

$$
\hat{\varphi}(x \xi)=e^{i \frac{g^{2}}{\bar{m}^{2}} \Delta_{\lambda}^{+}(\xi)}: e^{-i \frac{g}{\lambda} A(x+\xi)}: \psi(x)
$$

(A.8), (A.10) imply the commutator

$$
[\hat{\varphi}(x \xi) A(z)]=\frac{\lambda g}{\bar{m}^{2}}\left(\Delta_{\lambda}(x-z)-\Delta_{\lambda}(x+\xi-z)\right) \hat{\varphi}(x \xi) .
$$

Using (A.12) one finds

$$
\left(\Phi_{1}, \hat{\varphi}(x \xi) \Phi_{2}\right)=\left(\Phi_{1} \psi(x) \Phi_{2}\right)
$$

for physical state vectors

$$
\Phi_{1}, \Phi_{2} \in \mathfrak{F}, \quad \Phi_{1}, \Phi_{2} \in \hat{D}
$$

Let $\Phi_{1}^{\prime}, \Phi_{2}^{\prime}$ be vectors in $\mathfrak{H}^{\prime}$ of the form (A.11). For the matrix elements of $\hat{\varphi}(x \xi)$ between state vectors $\Phi_{1} \times \Phi_{1}^{\prime}, \Phi_{2} \times \Phi_{2}^{\prime}$ of the form (A.14) we find using (A.20)

$\left(\Phi_{1} \times \Phi_{1}^{\prime}, \hat{\varphi}(x \xi) \Phi_{2} \times \Phi_{2}^{\prime}\right)=\left(\Phi_{1}, \hat{\varphi}(x \xi) \Phi_{2}\right)\left(\left(\Phi_{1}^{\prime}, \Phi_{2}^{\prime}\right)+R(x \xi)\right)$

with

$$
\lim _{\xi \rightarrow 0} R(x, \xi)=0 \text {. }
$$

Hence the limit $\xi \rightarrow 0$ of (A.19) exists and yields

By

$$
\lim _{\xi \rightarrow 0}\left(\Phi_{1} \times \Phi_{1}^{\prime}, \hat{\varphi}(x \xi) \Phi_{2} \times \Phi_{2}^{\prime}\right)=\left(\Phi_{1}, \psi(x) \Phi_{2}\right)\left(\Phi_{1}^{\prime} \Phi_{2}^{\prime}\right)
$$

$$
\hat{\varphi}_{f}\left(\Phi \times \Phi^{\prime}\right)=\left(p \psi_{f} \Phi\right) \times \Phi^{\prime}
$$

the operator $\hat{\varphi}_{f}$ is defined on the linear span of vectors of the form (A.14). According to (A.23) the corresponding distribution $\hat{\varphi}(x)$ is the weak limit (A.17) for matrix elements between vectors of the form (A.14). For matrix elements between physical state vectors (A.22) it follows

$$
\left(\Phi_{1} \varphi(x) \Phi_{2}\right)=\left(\Phi_{1}, \psi(x) \Phi_{2}\right) \quad \Phi_{1}, \Phi_{2} \in \mathfrak{G}, \Phi_{i} \in \hat{D}_{i}
$$


Using again the commutation relations (A.8), (A.10) it can be shown that (A.17) is solved for $\psi$ by

(A.20) implies

$$
\psi(x)=\lim _{\xi \rightarrow 0}: e^{i \frac{g}{\lambda} A(x+\xi)}: \hat{\varphi}(x)
$$

Therefore

$$
[\hat{\varphi}(x), A(z)]=0 \text {. }
$$

$$
[\hat{\varphi}(x), p]=0 \text {. }
$$

One further obtains from (A.9), (A.10)

$$
[\hat{V}(x), A(z)]=0, \quad[\hat{V}(x), p]=0 .
$$

Hence the field operators

$$
\varphi(x)=\hat{\varphi}(x) p, V(x)=\hat{V}(x) p, \bar{\varphi}(x)=\bar{\varphi}(x) p
$$

leave $\mathfrak{G}$ invariant. Restricted to $\mathfrak{G}$ the domain of $\varphi_{f}, \bar{\varphi}_{f}$ and $V_{f}$ is

$$
D=\hat{D} \cap \mathfrak{H} \text {. }
$$

As has been pointed out by JAFFE and Symanzik [29] the Wightman functions of $\hat{\varphi}$ are not tempered distributions if the Wightman functions of $\psi$ are as seems to be the case in perturbation theory. For this reason it is advisable to allow for the spinor fields the more general concept of JAFFE $[3]^{19}$.

\section{(c) Dirac Equation in the Proca-Wentzel Formulation}

After these preparations we derive in the following the limit form of the Dirac equation of $\varphi$. Using (A.26) we obtain for matrix elements of (A.4) between physical state vectors

$$
\begin{aligned}
& \qquad i \gamma^{\mu} \partial_{\mu}\left(\Phi_{1}, \varphi(x) \Phi_{2}\right)=\lim _{\xi \rightarrow 0}\left(\gamma^{\mu} L_{\mu}(x \xi)+M_{0}(\xi)\left(\Phi_{1}, \varphi(x) \Phi_{2}\right)\right) \\
& L_{\mu}(x \xi)=\frac{1}{2}\left(\Phi_{1}, A_{\mu}(x+\xi) \psi(x) \Phi_{2}\right)+\frac{1}{2}\left(\Phi_{1}, A_{\mu}(x-\xi) \psi(x) \Phi_{2}\right) \\
& \text { We rearrange } \\
& \qquad \psi_{i} \in D_{i}, \quad D_{i}=\hat{D}_{i} \cap \mathfrak{S} .
\end{aligned}
$$

$$
\begin{aligned}
L_{\mu}(x \xi)= & \frac{1}{2}\left(\Phi_{1}, \hat{V}_{\mu}(x+\xi) \psi(x) \Phi_{2}\right)+\frac{1}{2}\left(\Phi_{1}, \hat{V}_{\mu}(x-\xi) \psi(x) \Phi_{2}\right) \\
& +\frac{\lambda g}{\bar{m}^{2}}\left(\Phi_{1}, \psi(x) \Phi_{2}\right) \partial_{\mu} \Delta_{\lambda}(\xi)
\end{aligned}
$$

by use of (5.12), (A.8) and (A.12). The last term vanishes for $\xi^{2}<0$, hence

$$
L_{\mu}=\frac{1}{2}\left(\Phi_{1},\left(V_{\mu}(x+\xi)+V_{\mu}(x-\xi)\right) \varphi(x) \Phi_{2}\right)
$$

19 If the Wightman functions of $A^{\mu}, \bar{\psi}, \psi$ are tempered distributions the operators may be defined for test functions $f \in \mathfrak{S}\left(R_{4}\right)$ or $\mathfrak{S}\left(R_{4}\right)$ on the domain generated by applying $A_{f}, \psi_{f}, \psi_{f}$ to $\Omega$. This domain, however, is not dense in the physical subspace $\mathfrak{G}$ necessarily $\varphi_{f}$ given by (A.25) is definable for the same class of test functions as $\psi_{f}$. 
because

$$
\left(\Phi_{1}, \hat{V}_{\mu} \psi \Phi\right)=\left(\Phi_{1}, p \hat{V}_{\mu} p \psi p \Phi_{2}\right)=\left(\Phi_{1}, V_{\mu} \varphi \Phi_{2}\right)
$$

Thus the limit form of the Dirac equation in the Proca formulation becomes

$i \gamma^{\mu} \partial_{\mu} \varphi(x)=\lim _{\xi \rightarrow 0}\left(g \frac{\gamma^{\mu}}{2}\left(V_{\mu}(x+\xi)+V_{\mu}(x-\xi)\right) \varphi(x)+M_{0}(\xi) \varphi(x)\right)$

for matrix elements

$$
\left(\Phi_{1}, \ldots \Phi_{2}\right) \quad \Phi_{i} \in D_{i} .
$$

Comparison with (A.4) shows that the form of the Dirac equation is invariant under the operator gauge transformation (5.12), (A.17).

\section{(d) Current Operator in Terms of Proca-Wentzel Fields}

In section 5 the limit form of the meson field equation in the Proca formulation was derived. It remains the problem to express the current

$$
j^{\mu}(x \xi)=p \hat{\jmath}^{\mu}(x \xi) p
$$

in terms of the Proca fields $V_{\mu}$ and $\varphi$. Using (5.12) the operator $\hat{Q}^{\mu}$ defined by (5.7) becomes

with

$$
\hat{Q}^{\mu}(x \xi \eta)=:\left(S e^{-i g \int_{x-\eta}^{x+\eta} d y^{\mu} \hat{V} \mu(y)} e^{i \frac{g}{\lambda}(A(x+\eta)-A(x-\eta))}\right):
$$

$$
\begin{gathered}
S=S_{A} S_{\varphi} \\
S_{A}=: e^{-i \frac{g}{\lambda} A(x+\xi)}:: e^{i \frac{g}{\lambda} A(x-\xi)}:=e^{\frac{g^{2}}{\lambda^{2}} \Delta^{+}(2 \xi)}: e^{-i \frac{g}{\lambda}(A(x+\xi)-A(x-\xi))}: \\
S_{\varphi}=\overline{\hat{\varphi}}(x+\xi) \gamma^{\mu} \hat{\varphi}(x-\xi) .
\end{gathered}
$$

With definition (5.8) and (A.10), (A.29), (A.30) one obtains

$$
\left(\Phi_{1}, \hat{Q}^{\mu}(x \xi \eta) \Phi_{2}\right)=e^{\frac{g^{2}}{\lambda^{2}} \Delta^{+}(2 \xi)} e^{2 \frac{g^{2}}{\lambda^{8}}\left(\Delta_{\lambda}^{+}(\xi-\eta)-\Delta_{\lambda}^{+}(\xi+\eta)\right)}\left(\Phi_{1}, Q^{\mu}(x \xi \eta) \Phi_{2}\right)
$$

for matrix elements (A.34) where $Q^{\mu}$ is given by (5.17). Hence relation (5.15) follows with

$$
\begin{gathered}
Z_{2}(\xi)=e^{\frac{g^{2}}{\lambda^{2}} \Delta^{+}(2 \xi)} Z_{2}^{\prime}(\xi) \\
Y(\xi \eta)=e^{-2 \frac{g^{2}}{\lambda^{2}}\left(\Delta_{\lambda}^{+}(\xi-\eta)-\Delta_{\lambda}^{+}(\xi+\eta)\right)} Y^{\prime}(\xi \eta) .
\end{gathered}
$$

Comparison of (5.5-7) with (5.15-17) shows that the form of the current operator is invariant under the operator gauge transformations (5.12), (A.17) provided the renormalization functions $Z_{2}, Y$ are transformed according to (A.36-37).

I am grateful to Drs. Gasiorowicz, HaAg, Swieca, Symanzik, Wess, Wick, K. WILSON, ZUMINO and ZWANZIGER for interesting and helpful discussions. 
Notes added in proof. As will be shown in a forthcoming paper an additional term of the form $\varrho(\xi) \frac{\xi_{\mu} \xi_{\nu}}{\xi^{2}} \partial^{\mu} \partial^{\nu} A(x)$ should be included in (1.1). Equ. (1.6) is then modified accordingly. I am grateful to Dr. K. WILson for having pointed this out to me.

Terms of the form $\varrho_{k} \frac{\xi_{\mu} \xi_{v}}{\xi^{2}} \partial^{\mu} \partial^{v} A$ should be included in (2.11-12). Equ. (2.14) is then obtained under the additional requirement.

\section{References}

1. Wightman, A. S.: Phys. Rev. 101, 860 (1956).

2. Garding, L., and A. S. Wightman: Arkiv Fysik 82, No. 2 (1965).

3. JAFFE, A.: Proc. MIT Conf. on Scattering Theroy, March 1966.

4. Valatin, J.: Proc. Roy. Soc. (London) Ser. A, 225, 535 and 226, 254 (1954).

5. Wilson, K. G.: Cornell Univ. Rep. 1964.

6. Brandt, R. J.: Ann. Phys. (N. Y.) 44, 221 (1967).

7. - Technical Report No. 673, University of Maryland.

8. Zimmermann, W.: Comm. Math. Phys. 6, 161 (1967).

9. - to be published.

10. Broido, M. M., and J. G. TAYlor: Phys. Rev. 147, 993 (1966), and references quoted therein.

11. Brandt, R. A., J. Sucher, and O. R. Woo: Phys. Rev. Letters 19, 801 (1967).

12. HAGEN, C. R.: Ann. Phys. (N. Y.) 31, 185 (1965).

13. Krold, N. M., T. D. Lee, and B. Zumino: Phys. Rev. 157, 1376 (1967).

14. Nishisima, K.: Phys. Rev. 133, B 204 (1964).

15. Jouvet, B.: Nuovo Cimento 3, 1132 (1965); 5, 1 (1957).

16. Johnson, K.: Nucl. Phys. 25435 (1961).

17. Symanzik, K.: unpublished.

18. WILson, K.: private communication.

19. Proca, J.: J. Phys. Radium 7, 347 (1936).

20. Wentzel, G.: Quantum theory of fields. New York: Interscience Publishers Inc. 1949.

21. Lee, T. D., S. Weinberg, and B. Zumino: to be published.

22. - , and B. ZuMrNo: to be published.

23. Schwinger, J.: Phys. Rev. Letters 3, 296 (1959).

24. Ogievetskir, O. V. I., and I. V. Polubarinov: J. Exptl. Theoret. Phys. (U.S.S.R.) 41, 247 (1961); 14, 179 (1962).

25. Feldman, G., and P. T. Matthews: Phys. Rev. 132, 823 (1963).

26. Stueckelberg, E. C. G.: Helv. Phys. Acta 11, 225 and 299 (1938).

27. FuJII, Y., and S. KAMEFUCHI: Nuovo Cimento 33, 1639 (1964).

28. Boulware, D. G.: Phys. Rev. 151, 1024 (1966).

29. JAFFE, A., and K. SymanzIK: private communication.

Prof. W. ZimmermanN

Courant Institute of

Mathematical Sciences

251 Mercer Street

New York, N.Y. 10012, USA 